\title{
REFLEXÕES E PRÁTICAS NA FORMAÇÃO DE PROFESSORES DE CIÊNCIAS NATURAIS
}

\section{REFLECTIONS AND PRACTICES IN TEACHER'S EDUCATION OF NATURAL SCIENCES}

\author{
Renato Barboza ${ }^{1}$ \\ Simone Alves de Assis Martorano ${ }^{2}$
}

\begin{abstract}
Resumo: A formação de professores de Ciências tem se mostrado com um dos grandes desafios da área de ensino nas últimas décadas. Isso decorre da sua complexidade. Formar um professor de Ciências apenas valorizando os aspectos pedagógicos da profissão ou, pelo contrário, valorizando apenas o conteúdo científico só fará com que a sua formação seja incompleta e insuficiente para lidar com os desafios do mundo atual. Assim, neste artigo apresentamos algumas reflexões sobre as práticas realizadas no nosso grupo com intuito de aprimorar a formação inicial de professores de Ciências. Apoiadas sobre a necessidade de existir uma base mínima de saberes inerentes à formação docente, estas práticas visaram trabalhar com aspectos relacionados com a atividade docente, seja os relacionados com o conhecimento dos conteúdos pedagógicos e contexto ou com os conhecimentos do conteúdo específico necessário à formação deste professor. Longe de apontar soluções, estes trabalhos vêm ressaltar a necessidade do trabalho integrado e aplicado em vários momentos da formação do estudante com o propósito de provê-lo de habilidades e competências que os auxiliarão em sua atuação profissional.
\end{abstract}

Palavras-chave: Ensino de Ciências; Ciências Naturais; Formação de Professores.

\begin{abstract}
The science Teacher education has been shown with one of the great challenges of the teaching area in the last decades. This is due to its complexity. To train a science teacher only by valuing the pedagogical aspects of the profession or, on the contrary, valuing only the scientific content will only make its education incomplete and insufficient to deal with the challenges of the world today. Thus, in this article we present some thought on the practices carried out in our group to improve the initial natural sciences teacher education. Based on the necessity to have a minimum of knowledge inherent in teacher education, these practices aimed to work with aspects related to the teaching activity, be they related to the knowledge of the pedagogical content and context or to the knowledge of the specific content necessary to the formation of this teacher. Far from pointing out solutions, these works highlight the necessity for an integrated and applied work in different moments of the student's education with the purpose of providing him with skills and competences that will assist them in their professional performance.
\end{abstract}

Keywords: Science Teaching; Natural Sciences; Teacher's Education.

\section{Introdução}

É inegável que a formação de professores de Ciências Naturais é uma tarefa complexa que tem passado por mudança substanciais nos últimos 50 anos (BAROLI;

\footnotetext{
${ }^{1}$ Doutor em Imunologia pela Universidade de São Paulo (USP). Professor adjunto da Universidade Federal de São Paulo (UNIFESP), Diadema, São Paulo, Brasil. E-mail: renato.rbz@gmail.com

${ }^{2}$ Doutora em Ensino de Ciências, Universidade de São Paulo (USP). Professora adjunta da Universidade Federal de São Paulo (UNIFESP), Diadema, São Paulo, Brasil. E-mail: sialvesmartorano@ @mail.com
} 
VILLANI, 2013). Essa complexidade decorre da necessidade de se considerar o desenvolvimento de uma série de competências durante a formação deste professor que permeiam, acima de tudo, o conhecimento da matéria a ser ensinada e dos processos de ensino aprendizagem, o questionamento sobre ideias científicas presentes no senso comum, a análise crítica do ensino tradicional, o preparo de atividades didáticas que promovam uma aprendizagem efetiva; além do saber avaliar, dirigir e tutorar o trabalho dos alunos (CARVALHO, 2013). Soma-se ainda, a necessidade dos futuros professores serem capazes de manterem-se atualizados com as mais recentes descobertas científicas. Por esses motivos, essa não é uma tarefa trivial, mas sim uma tarefa que exige do formador o constante pensar e repensar sobre o que deve ser ensinado.

Por essas razões, a formação de professores não é um ato de capacitação técnica e, por isso, não se limita às metodologias de ensino nem aos estudos dos conteúdos específicos, mas deve estar fundamentado em formação teórica consistente, interdisciplinar e contextualizada ética e socialmente (SILVA; BASTOS, 2012). Ainda, soma-se a este quadro as tensões decorrentes de diferentes pontos de vistas e da implementação de políticas educacionais que procuraram pautar o ensino de ciências nas últimas décadas (BAROLI; VILLANI, 2013). Diante disso, mesmo com uma grande quantidade de estudos sobre formação de professores, questionamentos como o feito por Graça em 1997, sobre o que um professor necessita saber, ainda têm sido feitos.

Proposições sobre a existência de uma base de saberes inerentes à formação docente são realizadas desde a década de 1980. Pioneiro nestes estudos, Shulman (1987), propôs uma base que leva em conta diversos conhecimentos, incluindo o conhecimento específico do conteúdo, o conhecimento do conteúdo curricular, o conhecimento pedagógico geral e do conteúdo, o conhecimento do contexto, dos estudantes e suas características, dos objetivos e finalidades, além dos conhecimentos sobre fundamentos filosóficos e histórico dos conteúdos. Estes conhecimentos, de acordo com Shulman, são o que diferenciam o professor de uma determinada disciplina de um especialista desta mesma disciplina. Baseado nestas proposições, Grosman (1990), sistematizou esta base de conhecimento, descrevendo num modelo amplamente estudado e adaptado (FERNANDEZ, 2015).

A sistematização de Pamela L. Grossman, elenca quatro eixos fundamentais como base para a construção dos saberes necessário ao docente, sendo eles o conhecimento do conteúdo pedagógico que é central e que se relaciona por lado com o conhecimento do conteúdo específico e do conhecimento pedagógico geral e do outro, conhecimento do 
contexto do em que o estudante está inserido (Figura 1). Tendo como base está sistematização, neste artigo elencaremos alguns trabalhos que temos realizado no curso de Licenciatura em Ciências da Universidade Federal de São Paulo que se integram a estes conceitos e buscam apresentar aos estudantes um ensino que atenda às exigências atuais da educação.

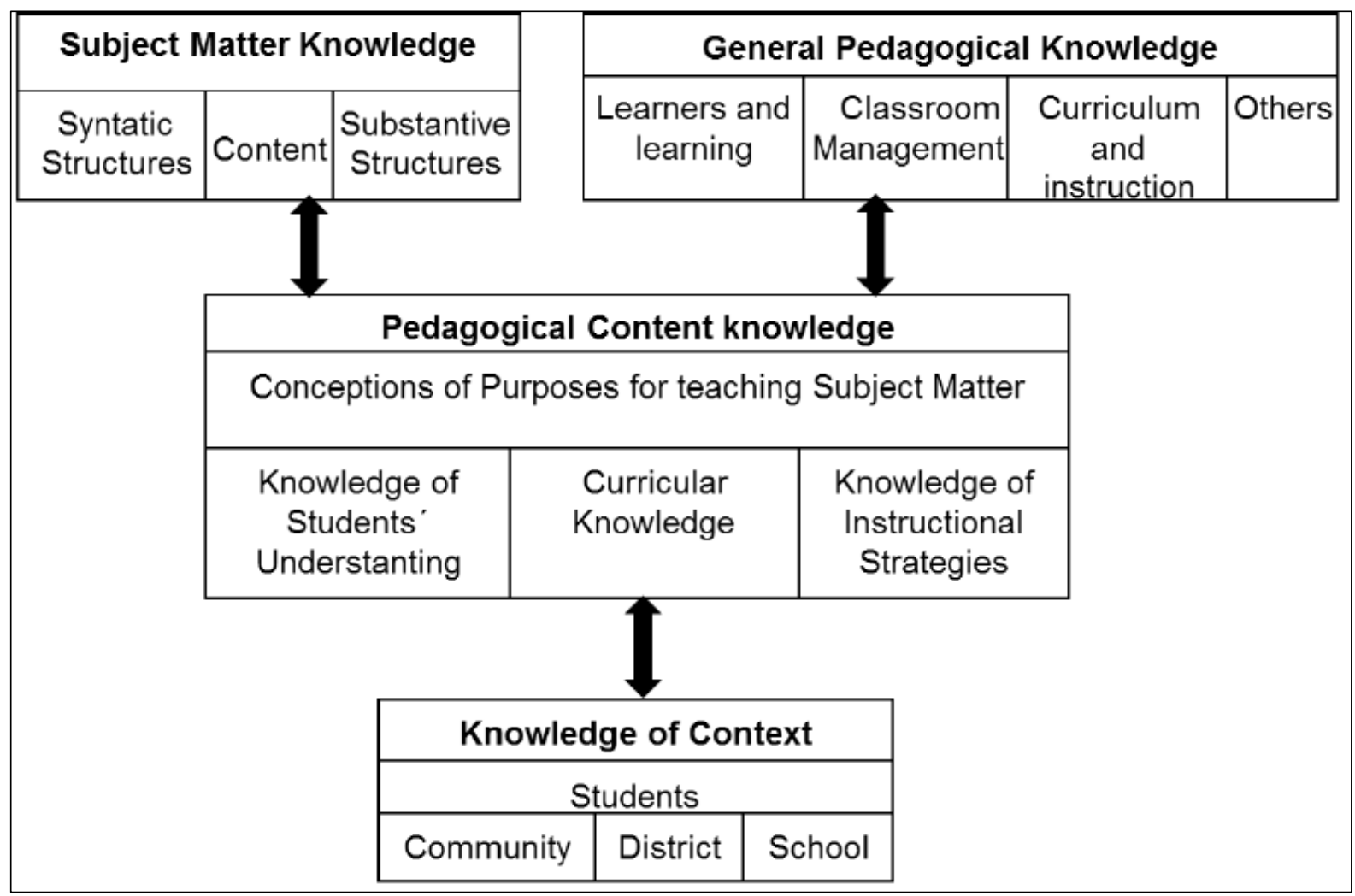

Figura 1: Modelo de relações entre os saberes docente proposto por Grossman (1990)

Fonte: Adaptado de (GROSSMAN, 1990, p.5)

\section{Sobre o curso de Licenciatura em Ciências da Universidade Federal de São Paulo}

O curso de Licenciatura em Ciências da Universidade Federal de São Paulo iniciou em 2010 e tem como principal objetivo a "busca por projeto de formação em Ciências alicerçado na integração de diferentes áreas do saber" (GOUW, 2017, p. 19). Com uma equipe formada por docentes das áreas de Ciências Naturais, Humanas e Matemáticas, o curso visa "formar um educador capaz de atuar nas diferentes realidades educacionais, inserindo-se profissional e singularmente nelas, de modo a transformá-las a partir de suas especificidades, em direção a um ideal de educação democrática, libertadora transformadora" (UNIFESP, 2014, p. 17). 
Para isso, ele conta com um currículo segmentado em duas partes. A primeira, formada por núcleo comum, que visa a formação do professor de Ciências para o Ensino Fundamental. Já a segunda está direcionada para formação de professores de Biologia, Química, Física ou Matemática para o Ensino Médio. Com isso, o curso proporciona um "forte perfil interdisciplinar, de modo a garantir uma formação sólida nos anos iniciais nas cinco áreas presentes em sua formação [...], mas com um diferencial de haver um direcionamento para uma área específica nos anos finais (UNIFESP, 2014, p. 14).

Além dos conteúdos relativos às ciências, os estudantes também têm uma considerável carga de disciplinas didáticas e pedagógicas, além de, como previsto para as Licenciaturas, às cargas horárias previstas para o Estágio Curricular Supervisionado ${ }^{3}$.

\section{Sobre os Estágios Supervisionados}

Como apontado por Pimenta e Lima (2012, p.24), a finalidade do Estágio Curricular Supervisionado, é "integrar o processo de formação do aluno, futuro profissional, de modo a considerar o campo de atuação como objeto de análise, de investigação e de interpretação crítica, a partir dos nexos com as disciplinas do curso". Sob esse ponto de vista, o estágio vem proporcionar a aproximação da realidade profissional. Este contato com a realidade também é fundamental para o estudante ganhar confiança, poder relacionar a teoria com prática, para aprender a lidar com os estudantes do ensino básico e, até mesmo, para aprimorar o conhecimento dos assuntos específicos de sua formação (AZEEM, 2011). No entanto, não haverá nenhum proveito se durante esse momento os professores em formação inicial não perceberem a importância do estágio.

Soma-se a este quadro o fato de que o Estágio Supervisionado per se abarca vários aspectos fundamentais da base para a construção dos saberes necessário ao docente, como proposto por Grossman (1990), visto que para sua realização a contento é necessário um certo domínio do conhecimento do conteúdo pedagógico, do conhecimento do conteúdo específico e do conhecimento pedagógico geral. Além de ser o espaço para a tomada de conhecimento do contexto escolar (conhecimento do contexto). Nesse sentido e reconhecendo as nuances do trabalho docente na formação inicial, realizamos um

\footnotetext{
${ }^{3}$ Para um maior detalhamento do curso, ver (KLUTH, 2017).
} 
levantamento para avaliar quais as percepções dos estudantes sobre este momento da sua formação.

Nosso estudo, embora pontual, auxilia na condução do trabalho do docente formador, pois evidencia as visões que os estudantes têm do Estágio Supervisionado, dentre elas, a ratificação pelo estudante de que este momento de formação tem sua importância explicita (BARBOZA; MARTORANO, 2015). Ao detalharmos nossa análise verificamos que essa importância se divide entre ser "importante do ponto de vista processo de formação inicial de professores" e ser "importante para que o licenciando tome contato com a realidade da escola pública de ensino básico”. Foi interessante notar, no entanto, que poucos estudantes tem a percepção de que o estágio pode servir como uma conexão entre a teoria aprendida na universidade e sua aplicação no ensino básico, interpretando o estágio como sendo teoria e prática, como proposto por Pimenta e Lima (2012).

Ainda, vimos uma clara preocupação do estudante sobre os aspectos práticos da disciplina, o que levou ao questionamento sobre a capacidade da maioria dos estudantesestagiários em fazer uma reflexão crítica sobre a sua atuação no estágio. No entanto, o fato da maioria dos estudantes-estagiários estarem mais preocupados com os aspectos práticos (calendário acadêmico, cumprimentos de horas de estágios, facilidade ou não de acesso às escolas, etc.), apenas revela suas preocupações momentâneas. Por isso, esses resultados evidenciam a necessidade do trabalho formativo não só no momento do estágio, mas durante todo curso.

\section{Formação integrativa}

Aproveitar o momento do estágio para realização de atividades complementares à formação, também é uma forma de aprimorar a formação do aluno. Por isso, realizamos um estudo que visou avaliar o uso da metodologia de Aprendizagem Baseada na Resolução de Problemas (ABRP) durante a realização do estágio supervisionado (MARTORANO; BARBOZA, 2015). A escolha dessa metodologia foi devido ao fato de que o seu desenvolvimento em sala de aula possibilita a discussão de importantes aspectos de um determinado tema, não se restringindo apenas aos conceitos científicos. Ainda que o ensino de Ciências da Natureza através da resolução de problemas é uma abordagem consistente com as recomendações dos Parâmetros Curriculares Nacionais (BRASIL, 1999), pois diversos conceitos e habilidades são aprendidos nesse contexto. Dentre estes, 
destacamos que a ABRP, ao modificar os processos de ensino e alterar a posição do professor na sala de aula, possibilita ao estudante ter uma maior autonomia, enquanto que o professor passa a ter um papel de mediador da aprendizagem do aluno (ESTEVES; COIMBRA; MARTINS, 2005).

No trabalho, divido em fases, apresentamos a proposta aos estudantes que, então, elaboram, seguindo a proposição de Esteves, Coimbra e Martins (2005) para elaboração de APRB, aulas direcionadas à resolução de questões do ENEM. Após a elaboração, os estudantes "praticaram" as aulas, apresentando-as para os professores orientadores do estágio. Ao final, eles aplicaram a metodologia na escola básica e fizeram uma avaliação sobre o processo. Os resultados oriundos deste estudo mostraram que os estudantes tiveram uma boa percepção das dificuldades relacionadas com a elaboração desta metodologia. Dentre as principais críticas, podemos citar o fato de que maioria achou uma metodologia difícil de ser desenvolvida nas escolas de ensino básico, mas ao mesmo tempo vários relatos apontam que a atividade foi satisfatória. Um ponto interessante a ser levantado foi a autocrítica dos estudantes, pois muitos classificaram sua atuação frente a aplicação como insatisfatória. De qualquer forma, os relatos nos deixam claro que todo o processo de elaboração e aplicação da metodologia proporcionou aos estudantes oportunidade de reflexão sobre a sua própria prática.

Em conjunto, esses dados evidenciam a necessidade de um acompanhamento do estudante de licenciatura durante o seu curso, no sentido de fazer com que ele conheça e entenda o contexto escolar em que irá atuar. Além disso, mostra ao docente formador quais as lacunas de formação licenciando possuem.

\section{O trabalho com os conteúdos específicos}

A formação do professor de ciências deve além do aprimoramento pedagógico, também levar em conta uma sólida formação dos conteúdos específicos. Neste particular, concentrando apenas na formação de professores de Biologia para o Ensino Médio, temos um problema atual que é a grande quantidade de conteúdo, visto que a Biologia contemporânea deixou de ser reducionista e tornou-se mais complexa, entendida agora como um complexo de sistemas interconectados (WOESE, 2004; GOLDENFELD; WOESE, 2007). Essa complexidade pode ser percebida no momento que se entende que os conhecimentos biológicos não são uma mera coleção de fatos, mas sim resultado de um processo investigativo intenso. No entanto, essa visão não está presente no ensino, 


\title{
$\eta_{\text {eBECEM }}$
}

que ainda apresenta uma fragmentação de seus conteúdos, prejudicando o seu aprendizado.

Como apontado por Carvalho (2016), existem uma exagerada quantidade de conteúdos de Biologia no Ensino Médio. De acordo com o levantamento feito pelos autores, há cerca de 3300 novos termos de Biologia em uma coleção de livro didático voltado ao Ensino Médio. Com base nessa premissa, estes autores iniciam um debate sobre o tema sugerindo uma redução do número de conteúdo que leve em conta critérios não arbitrários de seleção, mas sim aqueles baseados em conceitos estruturantes, com apresentada por Raul Gagliardi (1996). Em linhas gerais os autores, sugerem que é possível reduzir os conteúdos "com base na distinção entre Biologia funcional e evolutiva, como proposta por Ernst Walter Mayr (1904-2005) e François Jacob (19202013), assim como na busca de conceitos estruturantes proposta por Gagliardi" (CARVALHO, 2016). Ainda, de acordo com os autores "ao demarcar os conceitos estruturantes de uma ciência, podemos estabelecer objetivos primordiais a serem almejados em seu ensino" (CARVALHO, 2016, p.70)

A busca por uma redução de conteúdo baseados nesses critérios levaria a uma melhor compreensão da Biologia pelo estudante. Há de se ressaltar que o artigo de Carvalho et al., (2011) também leva em conta que temos

\begin{abstract}
outros critérios a considerar e discutir, sem dúvida, como, por exemplo, aqueles relacionados a conteúdos do ensino de Biologia que sejam importantes para que o estudante se torne mais capaz de participar de maneira informada de processos sociais de tomada de decisão nos quais o conhecimento biológico tenha papel importante, a exemplo dos recentes debates em nosso país sobre o uso de células-tronco embrionárias na pesquisa cientifica (CARVALHO, 2011, p. 92).
\end{abstract}

Tal apontamento evidencia a complexidade do ensino de Biologia. No contexto atual do ensino, não basta que os conteúdos sejam melhores compreendidos, eles devem também ser significativos para a vida do estudante, tornando-os capazes de desenvolverem competências e habilidades para a prática plena da cidadania.

Nesse sentido, Santos e Infante-Malachias já questionavam:

\footnotetext{
"Quanto?" e "O quê?" deve ser ensinado aos futuros professores sobre as diferentes disciplinas para que consigam atuar como interlocutores entre os especialistas e os cidadãos? Ora, como esses professores conseguirão atualizarse diante da crescente expansão da produção de conhecimento? Como se manterão informados sobre as mudanças em, pelo menos, cinco campos de produção de conhecimento distintos (Física, Química, Biologia, Astronomia, Ciências da Terra)?" (SANTOS; INFANTE-MALACHIAS, 2008, p. 567).
}

Destes questionamentos decorrem duas problemáticas essências para o ensino de Biologia e, por conseguinte, para o ensino de Ciências: a quantidade de conhecimentos acumulados; e a 
necessidade de sua seleção. Sobre isso, o que podemos dizer é que se trata de uma preocupação antiga. Por exemplo, ao tratar do ensino de Botânica no final do século XIX, Ernest Waldfried Josef Wenzel Match (1838 - 1916) já discutia este assunto em seu livro Popular Scientific Lectures. Tendo escrito que:

Como pode a mente prosperar quando a matéria é empilhada sobre a matéria, e novas matérias são constantemente empilhadas sobre matérias velhas, indigestas? A questão aqui não é tanto a da acumulação de conhecimento positivo como de disciplina intelectual. Parece também desnecessário que todas os ramos devem ser tratados na escola, e que exatamente os mesmos estudos devem ser prosseguidos em todas as escolas (MATCH, 1898, p. 368).

Entendendo que acúmulo do conhecimento é fato notório na história humana, o que temos é um agravamento desse cenário. Uma maneira de se lidar com esta problemática é proporcionar aos professores em formação inicial vivencias educativas nas quais seja valorizado, além dos produtos do conhecimento, os instrumentos pelos quais esse conhecimento se concretiza. Uma das maneiras de se fazer isso é realizar em sala de aula a análise direcionada de artigos científicos. Além disso, faz se necessário prover os estudantes de meios para que eles sejam capazes de selecionar conteúdos de forma significativa, com o intuito de favorecer a aprendizagem de diversas habilidades, permitindo a contextualização e aplicabilidade desses conteúdos no cotidiano dos estudantes.

Para trabalhar maneiras de o estudante de licenciatura entrar em contato com a produção do conhecimento científico, utilizamos uma estratégia onde eles estudam artigos científicos por meio de uma atividade colaborativa e ativa (BARBOZA; MOREIRA; FERRARI, 2015). Nesta atividade, os estudantes realizam uma atividade pré-aula de leitura do artigo juntamente com um guia. Após, foi ministrado aos estudantes uma aula curta para fornecer a eles as informações mais relevantes e que eles ainda não tinham tido contato durante o curso. Por fim, foi realizado uma atividade em sala de aula onde os estudantes discutiam os artigos e apresentavam suas intepretações. Com isso, foi possível apresentar conceitos científicos aos estudantes, além de provê-los com conceitos biológicos mais recentes.

Ao analisar os resultados desta atividade, verificamos que os alunos de licenciatura se tornaram mais familiares com os procedimentos experimentais utilizados na geração de conhecimentos. A atividade também auxiliou no desenvolvimento do pensamento crítico. Um ponto interessante que a análise dos resultados nos trouxe foi que mais de $90 \%$ dos estudantes entenderam a atividade como importante para sua formação, porém as justificativas desta importância foram variadas (dados não publicados). Na 
maioria dos casos, a importância referia-se ao fato da atividade auxiliar no desenvolvimento de habilidades e competências para leitura técnica científica. Porém, dentre os estudantes que tiveram esta percepção, poucos relacionaram estas habilidades e competências à atividade docente. A maioria dos estudantes consideram que a atividade se correlaciona melhor com curso de bacharelado.

Do nosso ponto de vista, este é um aspecto que deve ser mais explorado ao longo das aulas, pois falta a compreensão que o desenvolvimento destas habilidades e competências são essenciais para a aquisição de novos conhecimentos e que isso está diretamente relacionado com a profissão docente. Afinal, se tivermos a prerrogativa de que o conhecimento científico se desenvolve ao longo tempo, pois a ciência é dinâmica, e que para uma aprendizagem significativa é necessário manter-se atualizado sobre o que é produzido, então necessitamos sim de professores que possuam a habilidade de adquirir o conhecimento por meio de suas fontes primárias. Neste sentido, métodos que se baseiam no encorajamento dos estudantes na construção do novo conhecimento e no desenvolvimento do pensamento científico devem ser estimulados nas aulas de graduação (HANDELSMAN et al., 2004; MILLER et al., 2008). Evidências de que o uso deste tipo de estratégia de aquisição de conhecimento é importante para melhoria da aprendizagem do estudante podem ser encontradas na literatura (KITCHEN et al., 2003; FREEMAN et al., 2007; WALKER et al., 2008).

O conhecimento ou "domínio" do conteúdo a ser ensinado é uma prerrogativa básica do exercício da função docente. Porém, "na medida em que consideramos o conhecimento como um instrumento simbólico, dominá-lo significa compreender a ‘instrumentalidade’ nele presente (SFORNI, 2012, p. 473). Nóvoa (1999, p. 18) reforça que "o que faz falta é integrar estas dimensões no cotidiano da profissão docente, fazendo com que elas sejam parte essencial da definição de cada um como professor/a”. Assim, o domínio do conhecimento específico tem de ser acompanhado da competência de seus destes conteúdos de forma significativa, contextualizada e aplicável ao cotidiano dos estudantes.

No entanto, para que este objetivo seja alcançado, é necessário compreender as razões pelas quais os professores em formação fazem suas escolhas. Mais uma vez, trabalhando com nossos estudantes de Licenciatura, procuramos avaliar os modos de seleção de conteúdos que estes estudantes entendem como efetivos para serem aplicados no Ensino Médio (BARBOZA; MOREIRA; FERRARI, 2017). 
A partir de uma simples questão que solicitava que os estudantes escolhessem patógenos representante de diferentes grupos de organismos pertencentes a uma lista de organismos patogênicos pré-estabelecida. No entanto, está escolha deveria ser guiada por um entendimento: por que é importante o ensino do organismo em questão para estudantes do Ensino Médio? Cabe ressaltar aqui que importância não foi pré-estabelecia, cabendo aos estudantes a livre escolha, mas que deveria ser justificada.

Foi interessante notar que as escolhas dos estudantes levaram em conta a relevância dos organismos para a vida dos estudantes do ensino médio. Além possível perceber uma certa interferência temporal na escolha, ou seja, embora presente na lista, patógenos que não estavam sob o foco da mídia não foram citados. Por exemplo, não se viu citações sobre o vírus Chikungunia, visto que o surto deste organismo aconteceu no ano seguinte ao da coleta de dados. Ainda, em se analisando as justificativas de escolha, verificamos uma total ausência do uso do conhecimento para provas de ingresso em universidades, como o ENEM. Isso demostra um certo comprometimento dos estudantes com uma visão de formação cidadã.

Por fim, um outro aspecto do nosso trabalho na formação inicial de professores de ciências é o de trabalhar com os estudantes aspectos sobre a História e Natureza das Ciências. Trabalhando com professores de ciências em formação inicial, desenvolvemos uma atividade didática para que fosse possível levantar suas concepções sobre aspectos da Natureza da Ciência (COLAGRANDE; ARROIO; MARTORANO et al., 2016).

Os achados deste trabalham mostram que não há uma única maneira de se pensar e discutir ciência, no entanto, fica claro que o estudo da Natureza das Ciências oferece contribuições relevantes para o seu entendimento. Além disso, ao analisar os pensamentos e visões dos professores em formação inicial sobre a construção da ciência verificamos que estudantes que anteriormente tiveram contato com disciplinas que contemplem aspectos da Filosofia, História e Natureza apresentaram uma visão muito mais clara sobre o tema. Embora esse resultado pareça óbvio, é de se ressaltar que o tema nem sempre tem sido trabalhado em cursos de formação de ciências.

Em conjunto, este trabalho traz alguns práticas e reflexões realizadas pelo nosso grupo com o propósito de contribuir com a formação de professores de ciências. Pelos resultados sumarizados, percebe-se que a complexidade na formação destes professores e enfatiza a necessidade de se trabalha com os estudantes em diversos momentos da sua formação. 


\section{Considerações finais}

Como apontado por Lankshear e Knobel (2004) a atividade profissional não pode ser encarada como uma atividade rotineira de realização de tarefas, mas sim deve ser encarada como as atividades realizadas por médicos e advogados que também necessitam de um conhecimento profissional e uma experiência acumulada que os permita atuar em situações específicas. Por isso, é necessário assumir os pressupostos de Gatti (2016) que apontam que a qualidade formativa de professores deve levar em consideração os seguintes aspectos: que o fato educacional é cultural; que o professor é imprescindível; que o núcleo do processo educacional é o aluno; que é preciso considerar a heterogeneidade cultural e social de professores e alunos; e que práticas educacionais institucionalizadas determinam a formação de professores.

Ainda, os professores de ciências precisam assumir uma condição de profissional de educação, sendo a sua formação apoiada tantos nas dimensões políticas e pedagógicas quanto científicas (MARANDINO, 2003). Este profissional precisa conhecer o contexto ocupacional e a natureza de sua profissão (NÓVOA, 1995) e que deve ser formado "de maneira a se apropriar da produção do conhecimento" em sua forma plena, tanto pedagógico quanto das especificidades de sua área (MARANDINO, 2003).

Por tudo isso, retomamos o que havíamos dito incialmente: é inegável que a formação de professores de Ciências Naturais é uma tarefa complexa. Neste artigo, por entendermos que está complexidade dever ser trabalha em diferentes momentos e modos, apresentamos alguns dos trabalhos que temos realizado na formação inicial de professores para ressaltar estes aspectos. Afinal,

A segurança com que a autoridade docente se move implica uma outra, a que
se funda na sua competência profissional. Nenhuma autoridade docente se
exerce ausente desta competência. O professor que não leve a sério sua
formação, que não estude, que não se esforce para estar à altura de sua tarefa
não tem força moral para coordenar as atividades de sua classe. Isso não
significa, porém, que a opção e a prática democrática do professor ou
professora sejam determinadas por sua competência científica. Há professores
e professoras cientificamente preparadas, mas autoritários a toda prova. O que
eu quero dizer é a incompetência profissional desqualifica a autoridade do
professor (FREIRE, 2011, p. 89).

\section{Referências}

AZEEM, M. Problems of Prospective Teachers During Teaching Practice. Academic Research International, Lodhran, v. 1, n. 2, p. 308-316, 2011. 
BARBOZA, R.; MOREIRA, R. S.; FERRARI, M. F. R. Using active learning strategies to enhance student's cognitive skill in science education. Washington: Society for Neuroscience, 2015.

BARBOZA, R. et al. Critérios de seleção de conteúdos de Biologia utilizados por professores em formação inicial. In: ENCONTRO NACIONAL DE PESQUISA EM EDUCAÇÃO EM CIÊCNIAS, 11, 2017, Florianópolis. Anais... Florianópolis: Universidade Federal de Santa Catarina, 2017. p. 1-8.

BARBOZA, R.; MARTORANO, S. A. A. Avaliação da compreensão dos licenciando sobre a importância do estágio supervisionado na formação professores de Ciências. In: ENCONTRO NACIONAL DE PESQUISA EM EDUCAÇÃO EM CIÊCNIAS, 10, 2015, Águas de Lindóia. Anais... Águas de Lindóia, 2015. p. 1-8.

BAROLI, E.; VILLANI, A. Meio século de tensões na formação de professores de ciências no brasil. In: CONGRESO INTERNACIONAL SOBRE INVESTIGACIÓN EN DIDÁCTICA DE LAS CIENCIAS, 9, 2013, Girona. Anais... Girona: Universitat de Valencia, 2013. p. 286-290.

BRASIL. Ministério da Educação. Secretaria de Educação Média e Tecnológica. Parâmetros curriculares nacionais: ensino médio. Brasília: MEC/SEMTEC, 1999.

CARVALHO, A.M.P. Formação de professores de ciências: duas epistemologias em debate. In: CONGRESO INTERNACIONAL SOBRE INVESTIGACIÓN EN DIDÁCTICA DE LAS CIENCIAS, 9, 2013, Girona. Anais... Girona: Universitat de Valencia, 2013. p. 2784-2790.

CARVALHO, I. N. Uma proposta de critérios de selecionar conteúdos conceituais para o ensino médio de Biologia. 2016. 73 f. Dissertação (Mestrado em ensino, filosofia e história das ciências) - Insituto de Física, Universidade Federal da Bahia, Salvador, 2016.

COLAGRANDE, E. A.; ARROIO, A.; MARTORANO, S. A. A. Assessment on How Preservice Science Teachers View the Nature of Science. Journal of Turkish Science Education, v. 13, 2016, p. 293-307.

ESTEVES, E.; COIMBRA, M.; MARTINS, P. Aprendizagem da Física e Química Baseada na Resolução de Problemas: Um estudo centrado na sub-unidade temática "Ozonio na estratosfera", 2005. Disponível em:<http://www.enciga.org/files/boletins/61/a_aprendizagem_da_fq.pdf>. Acesso em: 13 nov. 2017.

FREIRE, P. Pedagogia da autonomia: saberes necessários à prática educativa. 43. ed. São Paulo: Paz e Terra, 2011.

GATTI, B.A. Formação de professores: condições e problemas atuais. Revista Internacional de Formação de Professores, Itapetininga, v. 1, n.2, p. 161-171, abr./jun. 2016.

GAGLIARDI, R. Los Conceptos Estructurales en el Aprendizaje por Investigación. Enseñanza de las Ciencias, Barcelona, v. 4, n. 1, p. 30-35, 1986.

GOLDENFELD, N.; WOESE, C. Biology's next revolution. Nature, v. 445, n. 7126, s.p. 2007.

GOUW, A. M. S. A prática pedagógica de Biologia no Curso de Ciências - Licenciatura. In: KLUTH, V. S. (Org.). Prática docente e formação de professores: reflexões à luz do ensino de Ciências. 1ed. São Paulo: Alameda, 2007. p. 131-147. 
GRAÇA, A. O conhecimento pedagógico do conteúdo no ensino do basquetebol. 1997. 331 f. Tese (Doutorado em Ciências do Desporto) - Faculdade de Desporto, Universidade do Porto, Porto, 1997.

GROSSMAN, P. L. The making of a teacher: teacher knowledge and teacher education. New York: Teachers College Press, 1990.

HANDELSMAN, J. et al. Scientific teaching. Science, v. 304, n. 5670, p. 521-522, 2004.

KLUTH, V.S. (Org.). Prática docente e formação de professores: reflexões à luz do ensino de Ciências. 1ed. São Paulo: Alameda, 2017.

LANKSHEAR, C.; KNOBEL, M. New literacies: Everyday practices and classroom learning 1nd ed. Buckingham, U.K.: Open University Press, 2004.

MATCH, E. Popular Scientific Lectures. Chicago: The Open Court Publishing CO, 1898.

MARANDINO, M. A prática de Ensino nas Licenciaturas e a pesquisa em ensino de ciências: questões atuais. Caderno Brasileiro de Ensino de Física, Florianópolis v. 20, n. 2, p. 168-193, ago. 2003.

MARTORANO, S. A. A.; BARBOZA, R. Aprendizagem Baseada na Resolução de Problemas: uma análise da metodologia na disciplina de estágio supervisionado no curso de ciências licenciatura. In: JORNADAS INTERNACIONALES "PROBLEMÁTICAS EN TORNO A LA ENSEÑANZA EN LA EDUCACIÓN SUPERIOR. DIÁLOGO ABIERTO ENTRE LA DIDÁCTICA GENERAL Y LAS DIDÁCTICAS ESPECÍFICAS”, 2, 2015, Luján. Anais... Luján, 2015, s.p.

MILLER, S. et al. The pipeline. Scientific teaching in practice. Science, n. 5906, v. 322, p. 1329-1330, 2008.

NÓVOA, A. Os professores na virada do milênio: do excesso dos discursos à pobreza das práticas. Educacão em Pesquisa, São Paulo, v. 25, n.1, p.11-20, jan./jun, 1999.

PIMENTA, S.G.; LIMA, M.S.L. Estágio e docência. São Paulo: Editora Cortez, 2012.

SANTOS, S.; INFANTE-MALACHIAS, M. E. Interdisciplinaridade e resolução de problemas: algumas questões para quem forma futuros professores de ciências. Educ. Soc., Campinas, v. 29, n. 103, p. 557-579, 2008,

SFORNI, M. S. F. Formação de professores e os conhecimentos teóricos sobre a docência. In: LIBÂNEO, J. C.; ALVES, N. (Org.). Temas de Pedagogia: diálogos entre didática e currículo. São Paulo: Cortez, 2012. p. 469-488.

SILVA, V. F.; BASTOS, F. Formação de Professores de Ciências: reflexões sobre a formação continuada. Alexandria, Florianópolis, v.5, n.2, p.150-188, set. 2012.

SHULMAN, L. S. Knowledge and teaching: foundations of a new reform. Harvard Educational Review, Harvard, v. 57, n. 1, p. 1-22, 1987.

UNIFESP. Projeto Pedagógico do Curso de Ciências - Licenciatura. 2014. Disponível em: $<$ http://www3.unifesp.br/prograd/app/cursos/index.php/prograd/arq_projeto/1225>. Acesso em: 21 dez.2016. 


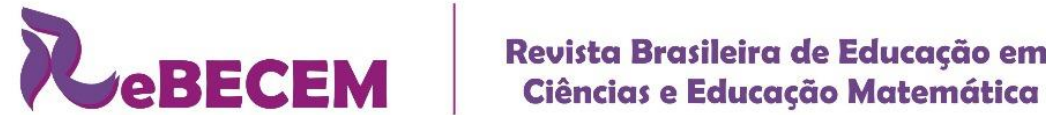

WOESE, C. R. A new biology for a new century. Microbiology and molecular biology reviews, New York, v. 68, p. 173-186, 2004.

Recebido em: 14 de novembro de 2017.

Aceito em: 11 de dezembro de 2017. 